\title{
Methanol coupling in the zeolite chabazite studied via Car-Parrinello molecular dynamics
}

\author{
CYNTHIA LO, CLAUDIU A. GIURUMESCU, \\ RAVI RADHAKRISHNAN and BERNHARDT L. TROUT* \\ Department of Chemical Engineering, \\ Massachusetts Institute of Technology, Cambridge, MA 02139, USA
}

(Received 6 July 2003; revised version accepted 1 December 2003)

\begin{abstract}
We have used Car-Parrinello constrained molecular dynamics to study the coupling of two molecules of methanol in the zeolite chabazite to form ethanol and water. We have chosen to study this reaction because it represents the formation of the first $\mathrm{C}-\mathrm{C}$ bond, which is thought to be the rate limiting step for the MTO and MTG processes. We have elucidated a new mechanism for this reaction that does not require the prior formation of surface methoxy groups or dimethyl ether intermediates. The mechanism involves stable intermediates of methane and protonated formaldehyde. We have also calculated an upper bound of the free energy barrier for the overall reaction, and found that it compares favourably with the rough experimental measurements available. Finally, we consider what are the natural reaction coordinates for the methanol-methanol coupling process.
\end{abstract}

\section{Introduction}

Zeolites are of major industrial importance as solid acid catalysts, being used throughout the petroleum and specialty chemical industry. They are microcrystalline silicon-oxide materials with various other species incorporated. For example, Brønsted acid sites are incorporated into the zeolite when certain atoms, such as $\mathrm{Al}$, are substituted for $\mathrm{Si}$ in the zeolite framework.

We are interested in studying coupling reactions of methanol in zeolites. From the industrial standpoint, such interest has been spurred from the possibilities of synthesizing olefins using methanol to olefin (MTO) processes and of developing more environmentally friendly processes for synthesizing gasoline using methanol to gasoline (MTG) processes. From the academic standpoint, it is of interest to understand the mechanism for the formation of the first $\mathrm{C}-\mathrm{C}$ bond, since it is thought that this is the rate limiting first step, along with the initial physisorption to the zeolite acid site, of the MTG and MTO reactions.

Because of the complexity of the reaction processes involving methanol in zeolites and of the limitations of applying experimental methods to these reactions, the reaction or reactions involving the formation of the first $\mathrm{C}-\mathrm{C}$ bond have never been isolated experimentally nor has a mechanism been definitively agreed upon. In fact, there have been more than 20 proposed mechanisms for the formation of the first $\mathrm{C}-\mathrm{C}$ bond [1]. One of these is

*Author for correspondence. e-mail: trout@mit.edu the oxonium ylide mechanism, where the first $\mathrm{C}-\mathrm{C}$ bond is formed from a trimethyl oxomium ion intermediate via either an intramolecular Stevens rearrangement or an intermolecular methylation. Others include formation of the first $\mathrm{C}-\mathrm{C}$ bond via insertion of carbenes or carbocations, catalysis $\mathrm{CO}$, and reaction via free radicals.

It is also thought, from four computational studies that address directly the formation of the first $\mathrm{C}-\mathrm{C}$ bond, that the formation of surface methoxy groups and/or dimethyl ether is a necessary first step towards the formation of ethanol and higher hydrocarbons. Blaszkowski and van Santen [2] conclude that the first $\mathrm{C}-\mathrm{C}$ bond is formed via reaction of a surface methoxy group with methanol or dimethyl ether and that pathways involving trimethyloxonium are not favourable. Tajima et al. [3] propose what they call the 'methane-formaldehyde mechanism' in which a methanol reacts directly with a surface methoxy species to form methane and formaldehyde as stable intermediates. These then react to form ethanol, which is dehydrated to ethylene. They found that their proposed pathway is more favourable than those incorporating oxonium ylide species, carbenes, or CO. Hutchings et al. [4] propose the interaction of a surface methoxy species with a second methanol molecule to form a surface ethoxy species, which after $\beta$-elimination forms ethylene. These three studies were all performed using small cluster models and static calculations. In the fourth study, Govind et al. [5] performed static calculations on 
a periodic model of two methanol molecules in ferrierite, and again proposed the reaction of a surface methoxy species with methanol or dimethyl ether to form ethanol or methyl-ethyl-ether; water does not play any visible role in their mechanism.

Despite the insight gained from these studies, they suffer from two major simplifications. First, the cluster calculations do not take into account the effects of the zeolite lattice including confinement in the channels and long-range interactions, and second, none of the studies take into account thermal and entropic effects caused by the dynamics of the motion of reactants and intermediates. In fact, the view of static intermediate or transition-state species can be only pictorial at best. In reality, the chemical species in the zeolite and the zeolite itself are constantly in motion and the form of the intermediates continually fluctuates.

We set out to simulate the coupling of $\mathrm{C}-\mathrm{C}$ from two methanol molecules around a Brønsted acid site in chabazite. We used sophisticated methods to address the time-scale problem, namely constrained dynamics and transition path sampling. We did not assume a priori intermediates or the form of the product with the $\mathrm{C}-\mathrm{C}$ bond.

\section{Methodology}

The zeolite we chose to study is chabazite, as shown in figure 1, which has been well characterized [6] and is active for the MTO and MTG processes [7]. We chose to study chabazite instead of ZSM-5, which is more commonly used in industry, because chabazite has the decisive computational advantage of having only 36 atoms per unit cell, making it tractable to treat periodically. We have used the unit cell and structural parameters provided by Smith et al. [6], and have included only one $\mathrm{Al}$ substituent per unit cell.

We used density functional theory [8-10], with the PW91 functional [11], to calculate electronic structure and energetics, and used a plane-wave basis set code

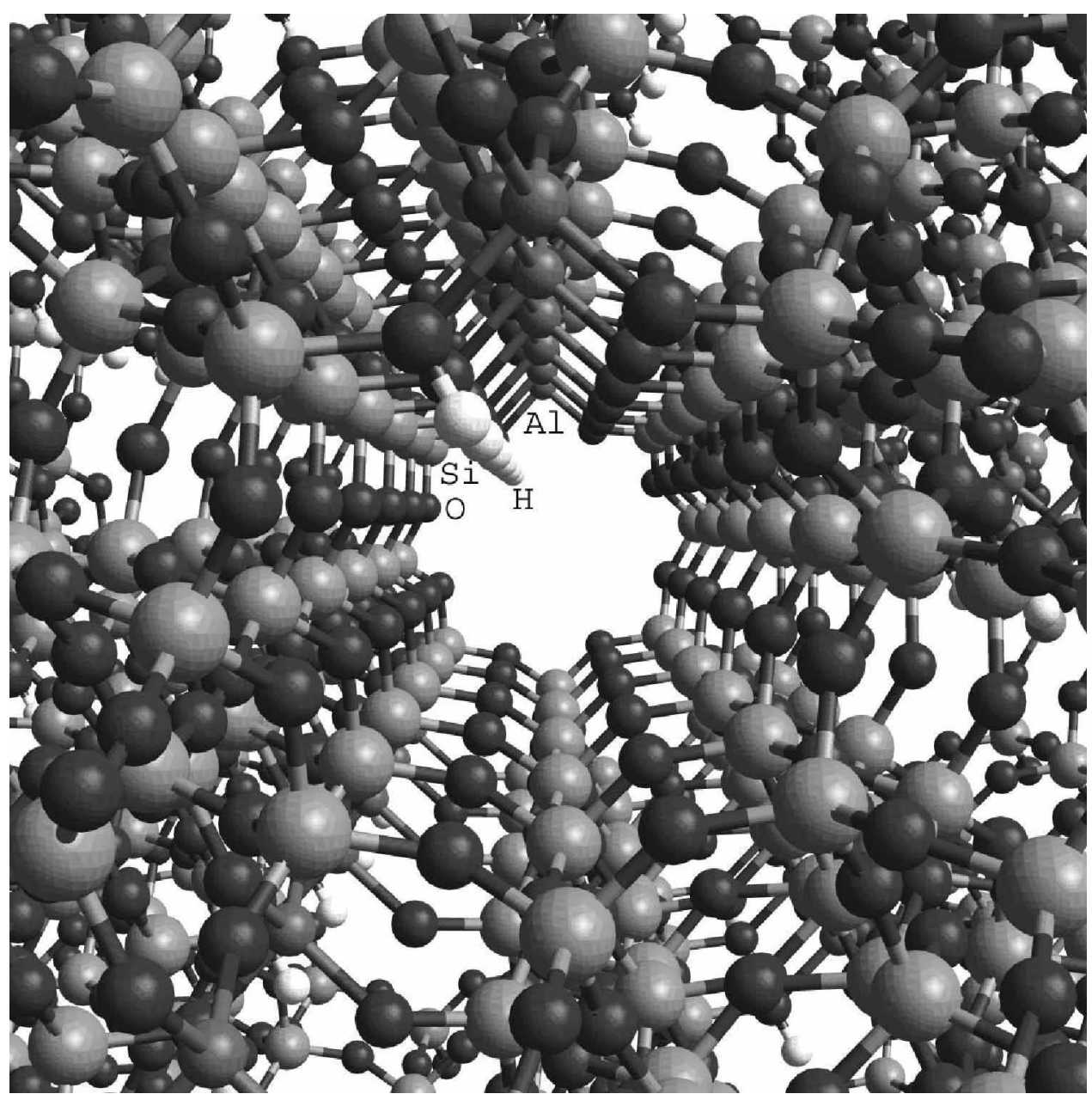

Figure 1. Perspective view of protonated chabazite with one $\mathrm{Al} /$ unit cell. 
with periodic boundary conditions [12] to model the zeolite as an infinite crystalline system. The plane-wave cutoff chosen was $55 \mathrm{Ry}$. This is very close to planewave cutoffs used by other researchers [13, 14], and we have found that it yields the gas-phase proton affinity of methanol to be $183.7 \mathrm{kcal} \mathrm{mol}^{-1}$, compared to $185.1 \mathrm{kcal} \mathrm{mol}^{-1}$ experimentally [15]. Only the $\Gamma$ point was sampled in the Brillouin zone. The fictitious electronic mass used was $1100 \mathrm{au}$, and timesteps of $7.0 \mathrm{au}(0.17 \mathrm{fs})$ were used for all runs.

We performed constrained molecular dynamics simulations of two methanol molecules in the chabazite unit cell, using the Car-Parrinello [16] approach. This method combines a quantum mechanical treatment of the electrons via density functional theory and a classical treatment of the nuclei, which are moved via a molecular dynamics simulation. All simulations were performed at $400^{\circ} \mathrm{C}$, the industrial operating temperature for the MTO and MTG processes [17]. We also note that the actual loading is 5-6 methanol molecules per active site in the MTG process [18], but we chose to study only two molecules per active site to simplify the interactions.

We wanted to simulate the $\mathrm{C}-\mathrm{C}$ bond formation process, so we chose the $\mathrm{C}-\mathrm{C}$ intermolecular distance as our reaction coordinate, and performed a series of non-zero temperature simulations to sample across that reaction coordinate. We first performed geometry optimizations at $0 \mathrm{~K}$ of the two methanol molecules in chabazite, and from the optimized structure, we decreased the $\mathrm{C}-\mathrm{C}$ distance and constrained its value. Initially, this decrease in distance occurred at $0.4 \AA$ intervals and closer to the transition state, it occurred at $0.2 \AA$ intervals. At each point along the reaction coordinate, we evaluated the ensemble averaged force due to the constraint along the constrained direction. From these simulations, we were able to compute the free energy as a function of the reaction coordinate by integrating the average force along the reaction coordinate [19-21]. A similar reaction coordinate was chosen for a study performed by Sauer et al. [22].

In order to determine properties averaged within the canonical ensemble, Nose-Hoover chain thermostat with a length of 4 and a characteristic frequency of $1500 \mathrm{~cm}^{-1}$ was used on the nuclear degree of freedom [23-25]. Each constrained molecular dynamics simulation run lasted $1.5 \mathrm{ps}$. During the first 0.5 picoseconds, the system was equilibrated and during the next picosecond, accumulated data were averaged to determine properties of interest. One picosecond of averaging was found to be enough to calculate the properties reported in this paper with small statistical uncertainties. Because the initial equilibration period was discarded, all references to time along the trajectory are made starting after this equilibration period.

\section{Results and discussion}

The overall reaction in the zeolite that we mapped is:

$$
2 \mathrm{CH}_{3} \mathrm{OH} \rightarrow \mathrm{CH}_{3} \mathrm{CH}_{2} \mathrm{OH}+\mathrm{H}_{2} \mathrm{O} \text {. }
$$

As mentioned in $\S 2$, we computed the free energy as a function of the reaction coordinate using equation (2) [23]:

$$
\frac{\mathrm{d} F}{\mathrm{~d} \xi}=\frac{\left\langle Z^{-1 / 2}[-\lambda+k T G]\right\rangle_{\xi}}{\left\langle Z^{-1 / 2}\right\rangle_{\xi}}=-\langle\lambda\rangle_{\xi},
$$

where $F$ is the free energy, $\xi$ is the value of the constraint, which in this case is the value of the $\mathrm{C}-\mathrm{C}$ distance, $\lambda$ is the Lagrange multiplier due to the constraint, which is equal to the negative of the force along the constrained $\mathrm{C}-\mathrm{C}$ direction, and $Z$ and $G$ are mass-weighted factors associated with the transformation from generalized to Cartesian coordinates. Because the constraint used in this study is a simple distance constraint, the expression can be simplified greatly to the term on the right-hand side of equation (2) [19]. Thus, the ensemble-averaged force on the constraint can be used to determine the free energy as a function of the reaction coordinate by integrating the average force along the reaction coordinate.

We plotted the free energy versus $\mathrm{C}-\mathrm{C}$ distance in figure 2. We set the free energy of the unconstrained system, where $\mathrm{C}-\mathrm{C}=5.14 \AA$, to be zero. We note that from figure 2 the free energy change needed for the $\mathrm{C}-\mathrm{C}$ distance to decrease from $5.14 \AA$ to $3.8 \AA$ is

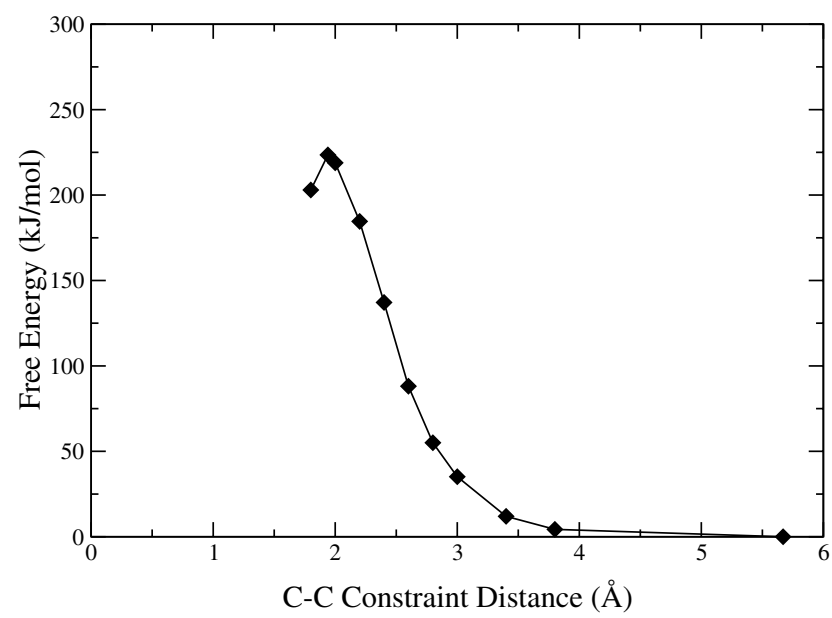

Figure 2. Free energy barrier for the methanol-methanol coupling reaction in the constrained ensemble $(T=673 \mathrm{~K})$. 
only $3.1 \mathrm{~kJ} / \mathrm{mol}^{-1}$, which would be rapidly and easily attained at $400^{\circ} \mathrm{C}$. We note that for $\mathrm{C}-\mathrm{C}=1.8 \AA$ we have already passed the peak of the free energy curve at $1.94 \AA$, so we can calculate the free energy barrier for the system to move across the constraint to be $\Delta F^{T S}=223.5 \mathrm{~kJ} / \mathrm{mol}^{-1}$.

To separate the energetic from the entropic terms in the free energy, we selected an equilibrated frame of the $\mathrm{C}-\mathrm{C}=2.0 \AA$ trajectory, reduced the bond length to $1.94 \AA$, and performed a geometry optimization. For reaction (1), this yields $\Delta U^{T S}(0 \mathrm{~K})=173.8 \mathrm{~kJ} \mathrm{~mol}^{-1}$, and $\Delta S^{T S}(673 \mathrm{~K})=-0.0738 \mathrm{~kJ}(\mathrm{~mol} \mathrm{~K})^{-1}$. Our calculated $\Delta U^{T S}(0 \mathrm{~K})$ is $10.0 \mathrm{~kJ} \mathrm{~mol}^{-1}$ lower than that found by Tajima et al. [3] and $77.2 \mathrm{~kJ} \mathrm{~mol}^{-1}$ lower than that found by Blaszkowski and van Santen [2]. The energetic term is also lower than the value obtained with mass spectrometry experiments on the most similar $\mathrm{C}-\mathrm{C}$ bond forming reaction we could find, where $212.26 \mathrm{~kJ} \mathrm{~mol}^{-1}$ of thermal energy was needed for the gas-phase reaction [26]:

$$
\left[\mathrm{CH}_{3} \mathrm{OHCH} 3\right]^{+} \rightarrow \mathrm{C}_{2} \mathrm{H}_{5}^{+}+\mathrm{H}_{2} \mathrm{O} .
$$

To the best of our knowledge, there exist only limited experimental values for free energy barriers and reaction rate constants for the $\mathrm{C}-\mathrm{C}$ bond forming process. Chen and Reagan [27] developed a kinetic model for olefin formation in ZSM-5:

$$
\begin{aligned}
& A \stackrel{k_{1}}{\rightarrow} B \\
& A+B \stackrel{k_{2}}{\rightarrow} B \\
& \frac{\mathrm{d} A}{\mathrm{~d} t}=-k_{1} A-k_{2} A B
\end{aligned}
$$

where $A$ represents the oxygenates and $B$ represents the olefins in the system. Using an autocatalytic assumption:

$$
\begin{aligned}
& A_{0}-A=B-B_{0} \Rightarrow A_{0}-A \approx B \\
& 1-\frac{A_{0}}{A}=1-\frac{1+\left(A_{0} / R\right)}{\left(A_{0} / R\right)+\exp \left(k_{2}(1+R) t\right)}
\end{aligned}
$$

where $R=\left(k_{1} / k_{2}\right)$, they determined that $k_{2}$ ranged in value between 0.1 and $1.1 \mathrm{~s}^{-1}$. These experiments were performed at $T=370^{\circ} \mathrm{C}$, so we can calculate their free energy barrier using transition state theory:

$$
\begin{aligned}
v & =\frac{k T}{h}=1.34 \times 10^{13} \mathrm{~s}^{-1} \\
K^{*} & =\frac{k_{2}}{v}=\text { between } 7.46 \times 10^{-15} \text { and } 8.21 \times 10^{-14} \\
\Delta F^{t s} & =-k T \ln K^{*}=\text { between } 161 \text { and } 174 \mathrm{~kJ} \mathrm{~mol}^{-1},
\end{aligned}
$$

where $v$ is the prefactor to the rate constant $k_{2}$, and $K^{*}$ is the equilibrium constant of the reaction. While this experimental range of $\Delta F^{t s}$ is lower than our calculated free energy barrier of $223.5 \mathrm{~kJ} \mathrm{~mol}^{-1}$, we note that ZSM-5 is known to be more active than chabazite for the MTO reaction [7], there may be a difference in reaction rates for olefin versus alcohol formation, and the kinetic model is highly simplified. Furthermore, our calculated value should be taken as an upper bound, considering, as shown below, that our simple constraint does not characterize the reaction process fully.

We show in figures 3 and 4 several snapshots along the $\mathrm{C}-\mathrm{C}=2.2 \AA$ and the $\mathrm{C}-\mathrm{C}=1.8 \AA$ constrained molecular dynamics trajectories. We see that in the $\mathrm{C}-\mathrm{C}=2.2 \AA$ trajectory, first a proton is transferred from the zeolite acid site to one of the methanol molecules, forming a methoxonium cation, which subsequently splits into methyl cation and water, breaking the $\mathrm{C}-\mathrm{O}$ bond. Then the remaining methanol transfers one of its protons to the methyl cation, forming methane and 'protonated formaldehyde'. We determined that these three intermediates are stable for at least $2.0 \mathrm{ps}$ by releasing the constraint and performing additional molecular dynamics simulations. In the $\mathrm{C}-\mathrm{C}=1.8 \AA$ trajectory, the water extracts a proton from methane. We then observe a concerted simultaneous transfer of a proton from $\mathrm{H}_{3} \mathrm{O}^{+}$to protonated formaldehyde, just as the latter transfers a proton back to the chabazite acid site, and the final formation of an ethanol-like species. Clearly ethanol cannot form since it has a $\mathrm{C}-\mathrm{C}$ distance of about $1.6 \AA$, but when we release the $\mathrm{C}-\mathrm{C}=1.8 \AA$ constraint, we do observe ethanol formation.

From the results of the constrained molecular dynamics simulations, we concluded that the process, which produces ethanol and water, involves stable intermediates of methane, protonated formaldehyde, and water, which would imply a two step reaction. However, from figure 2, it seems that there is only one transition state in the overall reaction. We thus needed to determine whether we were indeed sampling the transition state region or not.

For both the $\mathrm{C}-\mathrm{C}=2.2 \AA$ constrained trajectories, we calculated the committor probability distribution [28], where the committor probability $P_{B}$ represents the likelihood that trajectories originating in the assumed transition state region, with randomly chosen initial momenta, will reach the product state $B$ within a given time $\mathcal{T}$. Practically, we chose a set number of time slices equally spaced along these two trajectories, perturbed the initial momenta $p_{t}$ at each time slice $t$ by a small amount sampled from a Maxwell-Boltzmann distribution, and ran new unconstrained molecular dynamics trajectories forward (to $t=\mathcal{T}$ ) and backward (to $t=-\mathcal{T}$ ) in time. For this study we used 25 time slices 
and eight unconstrained molecular dynamics trajectories per time slice. At each $t$, all of the atoms in a given unconstrained molecular dynamics trajectory were randomly assigned positive or negative initial momenta to make the trajectories independent and uncorrelated with each other.

By the definition of Chandler and coworkers [28], at the transition state, the committor probability distribution should be unimodal and peaked at $P_{B}=0.5$. We plotted the distributions for the $\mathrm{C}-\mathrm{C}=2.2 \AA$ and the

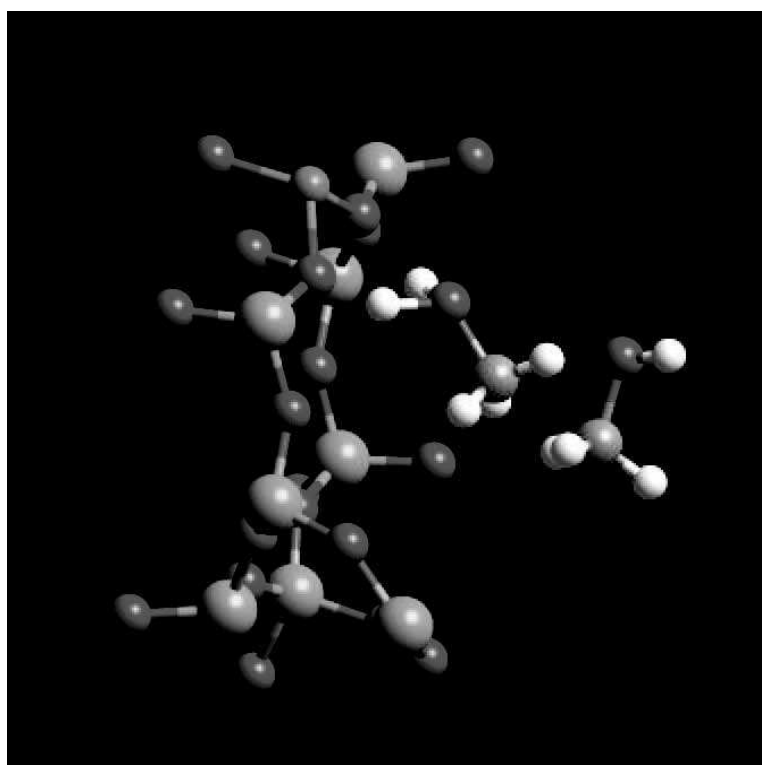

(a)

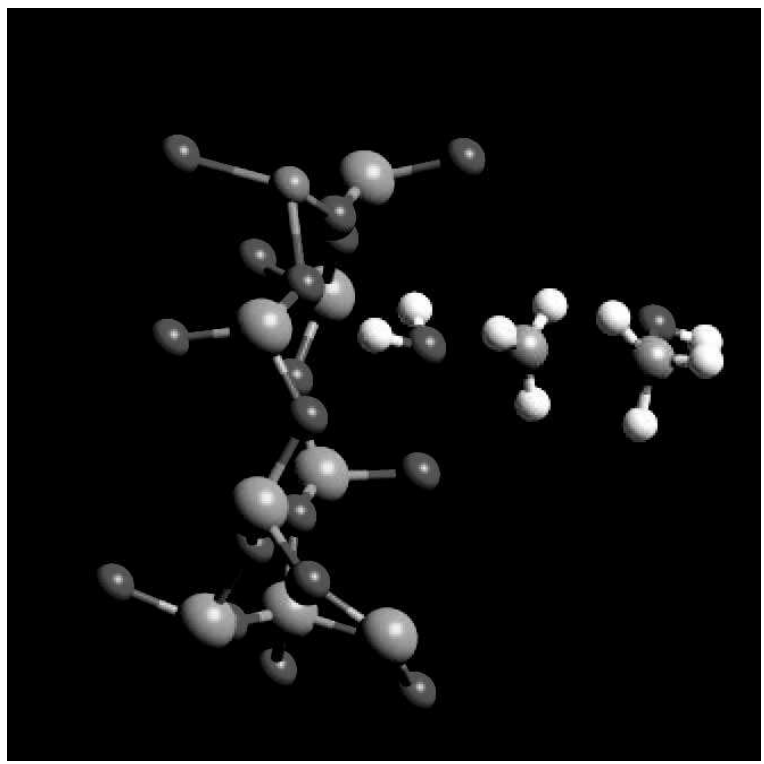

(c)
$\mathrm{C}-\mathrm{C}=1.8 \AA$ cases in figure 5 . The $\mathrm{C}-\mathrm{C}=2.2 \AA$ distance was chosen to see if we have properly captured the transition region for the formation of the methane and protonated formaldehyde species (figure 3), and the $\mathrm{C}-\mathrm{C}=1.8 \AA$ distance was chosen to see if we have properly captured the transition region for the formation of ethanol. We see that for the $\mathrm{C}-\mathrm{C}=2.2 \AA$ trajectory, the distribution is not unimodal, but in fact bimodal. The bimodal shape indicates that our chosen reaction coordinate does not govern the $\mathrm{C}-\mathrm{O}$ bond

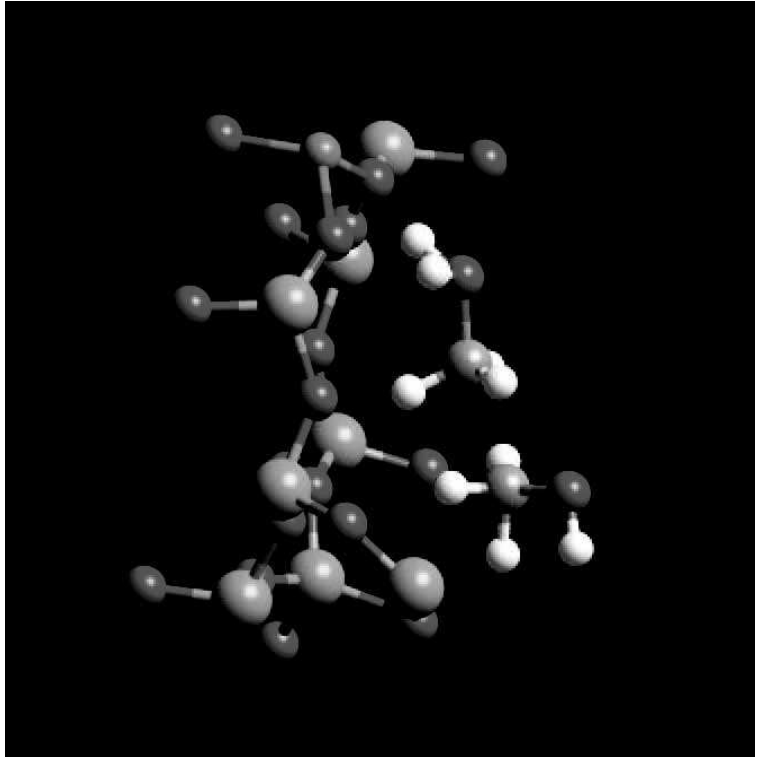

(b)

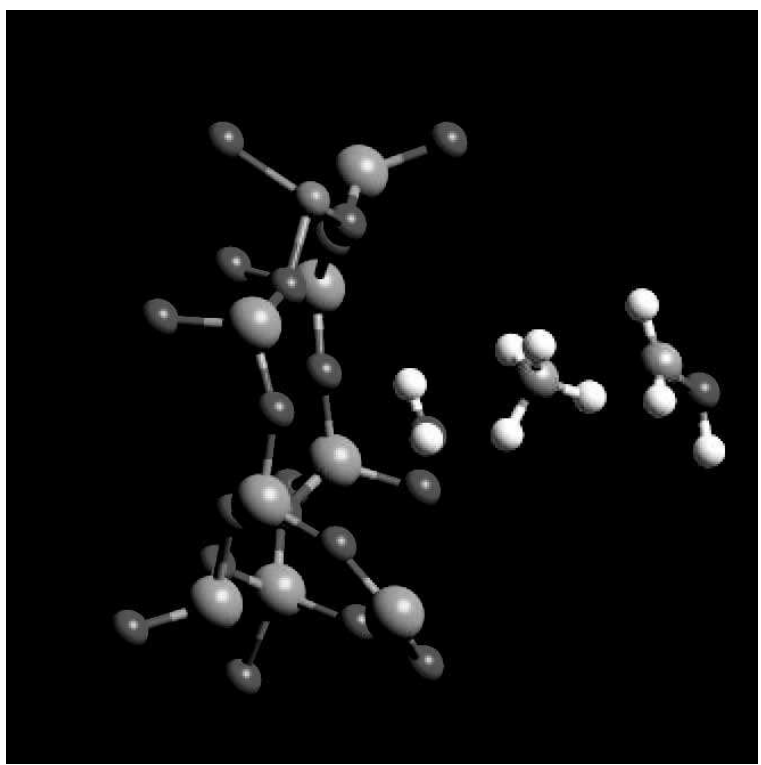

(d)

Figure 3. Snapshots from the $\mathrm{C}-\mathrm{C}=2.2 \AA$ constrained dynamics trajectory, showing: $(a)$ the initial physisorbed system, $(b)$ the protonation of methanol, $(c)$ the breaking of a $\mathrm{C}-\mathrm{O}$ bond to form water and methyl cation, and $(d)$ the extraction of a proton from the second methanol to form methane and protonated formaldehyde. 


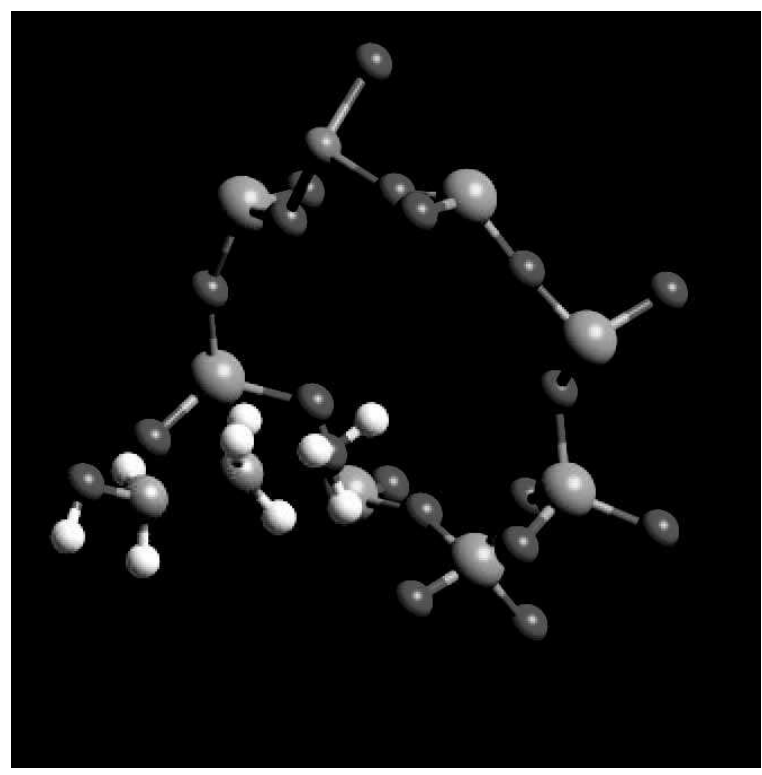

(a)

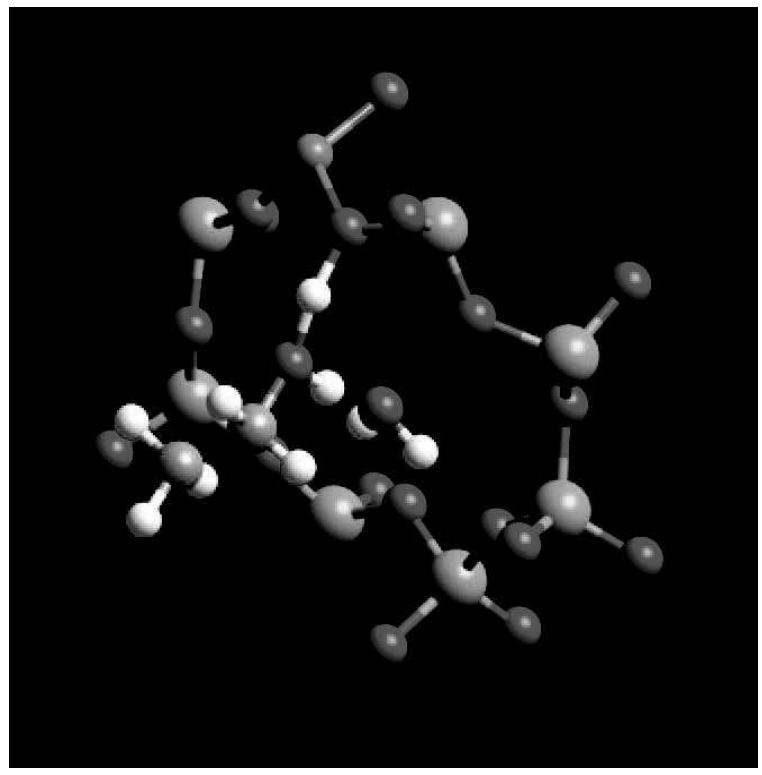

(b)

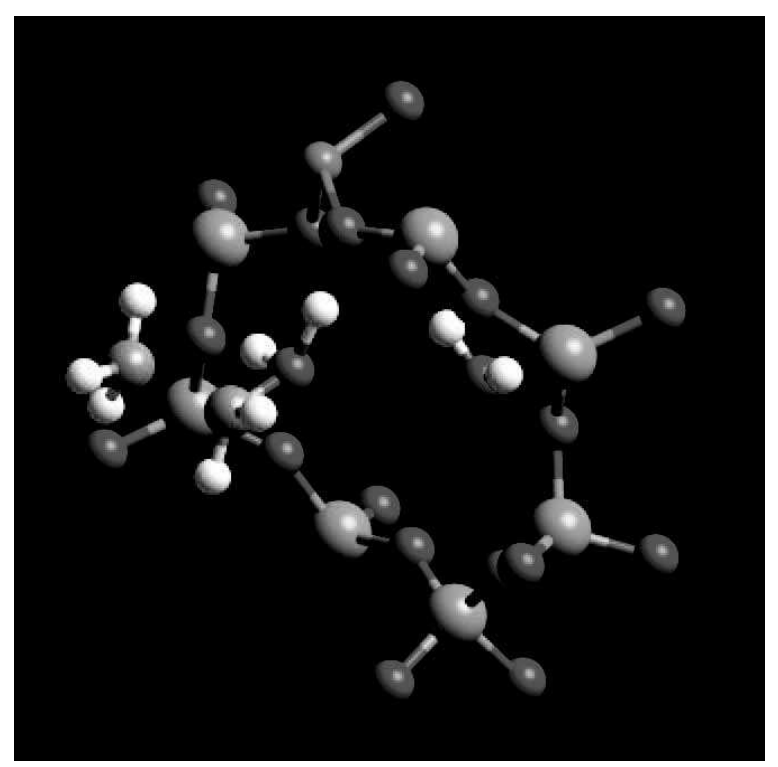

(c)

Figure 4. Snapshots from the $\mathrm{C}-\mathrm{C}=1.8 \AA$ constrained dynamics trajectory, showing: $(a)$ the stable intermediates, $(b)$ the concerted transfer of a proton from water to protonated formaldehyde to the zeolite, and $(c)$ the final ethanol-like product.

breaking process to form the intermediates [28]. The $\mathrm{C}-\mathrm{C}=1.8 \AA$ distribution is skewed and not unimodal, again indicating that we are not sampling the transition state region. However, the unconstrained trajectories corresponding to $P_{B}=0.5$ do show roughly the same $\mathrm{C}-\mathrm{O}$ bond breaking mechanism for the first step, and direct $\mathrm{C}-\mathrm{C}$ bond formation in the second step of the methanol-methanol coupling process. Furthermore, the constrained dynamics has given us initial dynamic trajectories that we have used to perform transition path sampling via Monte Carlo simulations in path space [29-31]. The transition path sampling has yielded similar reaction pathways to those in figures 3 and 4 . Nevertheless, the two committor probability distributions indicate that the $\mathrm{C}-\mathrm{C}$ distance reaction coordinate chosen by us and Sauer et al. [22] is not the correct coordinate for the methanol-methanol coupling process. We therefore need to determine the magnitude of the free energy barrier for both steps of the reaction using multiple reaction coordinates elucidated from the unconstrained $P_{B}=0.5$ trajectories. We are currently performing this work via constrained 


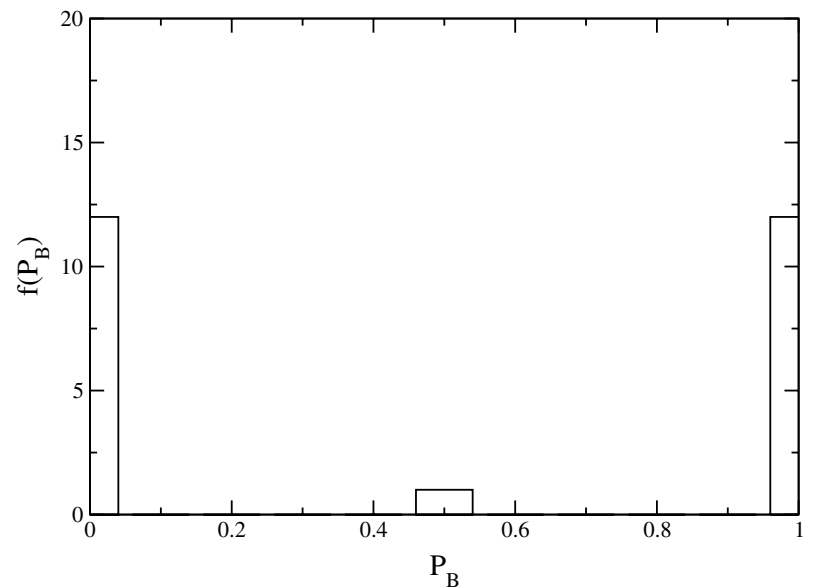

(a)

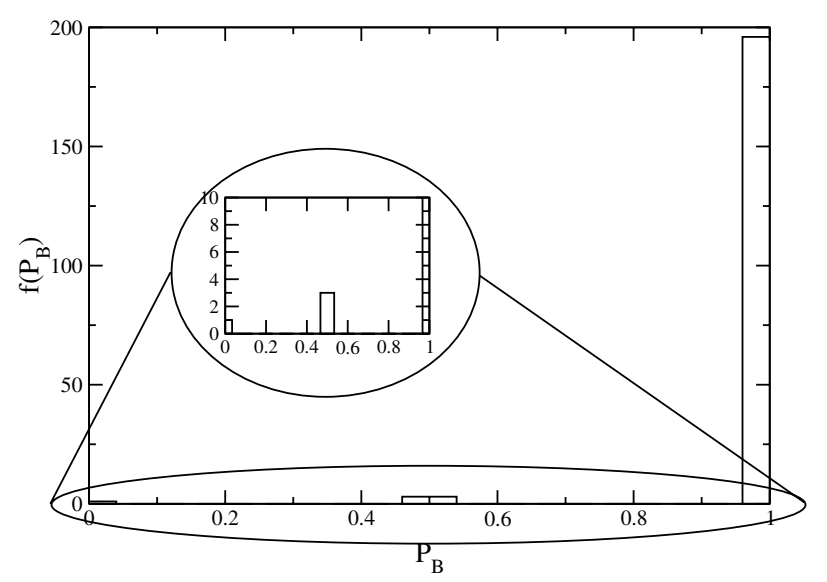

(b)

Figure 5. Committor probability distributions for the (a) $\mathrm{C}-\mathrm{C}=2.2 \AA$ and $($ b) $\mathrm{C}-\mathrm{C}=1.8 \AA$ constrained trajectories.

molecular dynamics and transition path sampling methods.

Our use of constrained molecular dynamics simulations has given us insight into the reaction process, initial dynamic trajectories, likely reactants and products, and an upper bound on the free energy barrier of reaction. Also, in our simulations, in contrast to the assumptions made by other researchers, we do not observe the formation of a surface methoxy species or a dimethyl ether intermediate.

\section{Conclusions}

We have elucidated, using Car-Parrinello molecular dynamics, a new mechanism for direct ethanol formation from methanol at $400^{\circ} \mathrm{C}$. This mechanism does not require the formation of surface methoxy groups nor a dimethyl ether intermediate, but does involve stable intermediates of methane and protonated formaldehyde. Our choice of using the $\mathrm{C}-\mathrm{C}$ distance as the reaction coordinate to be sampled was overly simplistic. Nevertheless, we did gain insight into the reaction process, in addition to an upper bound on the free energy barrier for $\mathrm{C}-\mathrm{C}$ coupling. The mechanism that we found is likely to correspond to a viable physical pathway because the upper bound of our activation energy is lower than that reported for other mechanisms $[2,3]$. The qualitative insight from our constrained dynamics calculations is consistent with the results of the more rigorous transition path sampling methods that we performed.

We would like to thank the National Science Foundation for a Graduate Research Fellowship and Grant (CTS-9984301) for financial support, the National Center for Supercomputing Applications for computing time, and the Sloan Foundation and the MIT UROP office for partial support.

\section{References}

[1] Stöcker, M., 1999, Micropor. Mesopor. Mater., 29, 3.

[2] Blaszkowski, S. R., and van Santen, R. A., 1997, J. Am. chem. Soc., 119, 5020.

[3] Tajima, N., Tsuneda, T., Toyama, F., and Hirao, K., 1998, J, Am. chem. Soc., 120, 8222.

[4] Hutchings, G. J., Watson, G. W., and Willock, D. J., 1999, Micropor. Mesopor. Mater., 29, 67.

[5] Govind, N., ANDZelm, J., Reindel, K., and Fitzgerald, G., 2002, Int. J. molec. Sci., 3, 423.

[6] Smith, L. J., Davidson, A., and Cheetham, A. K., 1997, Catal. Lett., 49, 143.

[7] Yuen, L.-T., Zones, S. I., Harris, T. V., Gallegos, E. J., and Auroux, A., 1994, Micropor. Mater., 2, 105.

[8] Hohenberg, P., and Kohn, W., 1964, Phys. Rev. B, 136, B864.

[9] Konn, W., and Sham, L. J., 1965, Phys. Rev. A, 140, A1133.

[10] PARr, R. G., and YAng, W., 1989, Density Functional Theory of Atoms and Molecules (New York: Oxford University Press).

[11] Perdew, J. P., 1991, Electronic Structure of Solids '91, edited by P. Ziesche and H. Eschrig (Berlin: Akademie Verlag), pp. 11-20.

[12] Hutter, J., Alavi, A., Deutsch, T., Bernasconi, M., Goedecker, S., Marx, D., Tuckerman, M., and PARRinello, M., 1995-1999, CPMD Version 3.3, MaxPlanck-Institut für Festkörperforschung and IBM Zurich Research Laboratory.

[13] Shah, R., Payne, M. C., and Gale, J. D., 1996, J. phys Chem., 100, 11688.

[14] Shah, R., Payne, M. C., Lee, M.-H., and Gale, J. D., 1996, Science, 271, 1395.

[15] Aue, D. H., and Bowers, M. T., 1979, Gas Phase Ion Chemistry, edited by M. T. Bowers, Vol. 2 (New York: Academic Press), 2, Chap. 9, pp. 2-52.

[16] Car, R., and Parrinello, M., 1985, Phys. Rev. Lett., 55, 2471. 
[17] KeIL, F. J., 1999, Micropor. Mesopor. Mater., 29, 49.

[18] Mirth, G., Lercher, J. A., Anderson, M. W., and Klinowski, J., 1990, J. chem. Soc., Faraday Trans., 86, 3039 .

[19] Carter, E. A., Ciccotti, G., Hynes, J. T., and Kapral, R., 1989, Chem. Phys. Lett., 156, 472.

[20] Sprik, M., and Ciccotti, G., 1998, J. chem. Phys., 109, 7737.

[21] Payne, M. C., Hytha, M., Stich, I., Gale, J. D., and Terakura, K., 2001, Micropor. Mesopor. Mater., 48, 375.

[22] Sauer, J., Sierka, M., and HaAse, F., 1999, Transition State Modeling for Catalysis, edited by D. G. Truhlar and D. Morokuma, ACS Symposium Series, Vol. 721 (Washington, DC: American Chemical Society), pp. 358-367.

[23] Nosé, S., 1984, Molec. Phys., 52, 255.
[24] Hoover, W. G., 1985, Phys. Rev. A, 31, 1695.

[25] Martyna, G. J., Klein, M. L., and Tuckerman, M., 1992, J. chem. Phys., 97, 2635.

[26] Smith, R. D., and Futtrel, J. H., 1976, Chem. Phys. Lett., 41, 64.

[27] Chen, N. Y., and Reagan, W. J., 1979, J. Catal., 50, 123.

[28] Bolhuis, P. G., Dellago, C., and Chandler, D., 2000, Proc. Natl. Acad. Sci. USA, 97, 5877.

[29] Bolhuis, P. G., Chandler, D., Dellago, C., and Geissler, P. L., 2002, Annu. Rev. phys. Chem., 53, 291.

[30] Bolhuis, P. G., Dellago, C., and Chandler, D., 1998, Faraday Discuss, 110, 421.

[31] Dellago, C., Bolhuis, P. G., and Chandler, D., 1998, J. chem. Phys., 108, 9236. 